\title{
Efficient RLC Macromodels for Digital IC Interconnect
}

\author{
Bogdan Tutuianu \\ Motorola Inc., Austin, Texas \\ bogdan@ibmoto.com
}

Daksh Lehther

Motorola Inc., Austin, Texas

daksh@ibmoto.com

Madhulima Pandey

Motorola Inc., Austin, Texas

madhu@ibmoto.com

\section{Ross Baldick}

The University of Texas at Austin

baldick@ece.utexas.edu

\begin{abstract}
The paper presents a new method to synthesize macromodels for very large on-chip interconnection networks which can be simulated very efficiently with traditional SPICE-like simulators. The method is taking advantage of the fact that in CMOS VLSI circuits, the receiving ports of the clock distribution network can be accurately modeled by lumped passive impedances. Our method simplifies the task of simulating the interconnect by building a reduced order macromodel only for the subset of driving ports of the net. The signals at the passive ports can be determined, in a later stage, as linear combinations of a reduced set of primary signals, obtained during the simulation of the driving ports macromodel. The simulation time for the macromodels generated in this way is greatly reduced, the size of the macromodels is kept small and the accuracy is preserved.
\end{abstract}

Keywords: simulation, macromodels, clock distribution networks 


\section{INTRODUCTION}

In modern microprocessors, due to higher operating frequencies, tight design constraints are imposed on critical nets such as the clock distribution networks. As a consequence, the designers need to perform a more detailed circuit analysis with an accurate SPICE-like circuit simulator, as opposed to relying on the traditional static timing analysis. At the same time, the size of the clock circuits that must be simulated has increased dramatically due to two factors: increased transistor and wiring density, and high operating frequencies. Increased transistor and wiring densities allow for more transistors to be packed on the same chip and, naturally, this results in much larger clock distribution networks. In order to capture accurately the large bandwidth of the signals, the distributed RLC representation of the interconnect also needs to be more complex. So, the designers are confronted with the fact that they have to design much larger structures and, for that, they have to use more and more accurate simulators, which are very slow. This makes the design of clock networks a very time consuming task and, for a faster design cycle time, new ways to improve things are needed. One such way to reduce the simulation time of very large circuit structures is to use reduced order macromodels for the RLC interconnect sections.

The problem of building macromodels for linear circuits is very well understood and not new. However, the problem of building macromodels is usually left at the point where a reduced model is obtained, usually in the frequencydomain form, and most of the effort is put into quantifying the accuracy and the stability of the reduced order model. One of the first attempts to generate macromodels that could be integrated with general purpose simulators is presented in [Kim 94]. The macromodels generated in [Kim 94] are based on AWE [Pillage 90] and, as a consequence, they are not guaranteed to be stable. Also based on AWE is the method proposed in [Dartu 96], which takes advantage of the fact that most of the ports (the receiving ports) can be modeled as passive loads and can be included in the macromodel, but it has the draw-back that can be used only for RC interconnect circuits. Another method to generate macromodels for simulation purposes is [Feldman 95], which uses the Padé-Via-Lanczos algorithm, thus resulting in more stable reduced order models, as compared to AWE. Other notable methods for generating reduced order approximations as well as lumped circuit models are [Kerns 96, Mangold 98]. A powerful new method is the coordinate-transformed Arnoldi algorithm [Silveira 96, Elfadel 97], which guarantees stable reduced order models. In our approach, we use an implementation of the Arnoldi algorithm, PRIMA [Odabas 97], which guarantees the passivity of the reduced order models.

In an industrial environment, several other problems, beside passivity and accuracy, can appear: how to use the macromodels with an existing simulator, 
how to build models which are reduced in size (preferably smaller than the initial circuit), how to simulate the macromodels in the most efficient way, how to store and post-process the simulation results, how to integrate the macromodels into the general design flow. Some of these "implementation issues" can have a direct negative impact on the entire macromodel generation algorithm and the best theoretical approach may not be the most feasible one in practice.

The use of frequency-domain reduced order models normally requires access to the core of the simulator, because inputting such models in file format to a SPICE-like simulator is prohibitive. One solution to this problem is to use synthesized lumped circuit macromodels based on the frequency-domain reduced order representation because lumped circuit elements are part of the input format of any commercial circuit simulator.

The benefit of "linearizing" receiving (sink) ports and including their passive loads into the interconnect [Dartu 96] is that the signal activity is fully characterized only by the driving port signals. So, with a macromodel built only for the subset of driving ports, we can fully capture the behavior of the interconnect, if the sink port loads are known a priori. But, in order to generate the signals at the sink ports, we have to post-process the simulation results for the macromodel corresponding to the driving ports. An efficient way to do that is to generate a reduced set of "primary signals" during the simulation of the macromodel, signals which are later re-used as construction blocks for any other signal of interest (sink port response) through simple linear combinations. As a consequence, the macromodel size is small (basically only the subset of driving ports) and the simulation time is greatly improved while the accuracy is preserved.

In Section 1 we describe the modified macromodel equations based on the admittance formalism. In Section 2 we describe in detail the actual macromodel building process and the problems associated with it. In Section 3, considerations on the simulation speed are discussed, while in Section 4 we present some performance statistics of a tool that has been developed based on our macromodeling method and is used at Motorola's PowerPC ${ }^{T M} 1$ microprocessor design center, Somerset Design Center in Austin, Texas.

\section{THE ADMITTANCE FORMALISM OF MULTIPORT STRUCTURES}

In the admittance $(Y)$ formalism [Valken 60] a sink port is "active" even when no current is flowing through the port because its effect at other ports is seen as a function of the voltage across the port pins. For a circuit with $p$ ports,

\footnotetext{
${ }^{1}$ PowerPC is a trademark of International Business Machines Corp. and is used under license by Motorola Inc.
} 

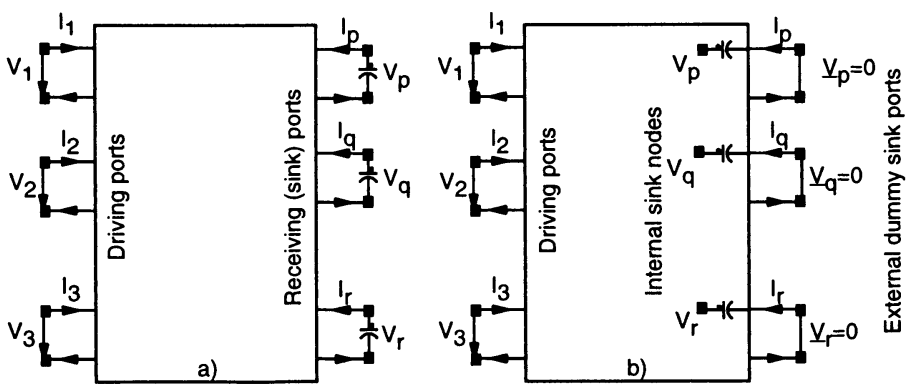

Figure 1 The multi-port representation: a) typical $Y$ formalism; b) modified $Y$ formalism.

$d$ drivers and $p-d$ sinks, the equation of port $j$ is given in (1.1):

$$
I_{j}(s)=V_{1} \cdot Y_{1 j}+V_{2} \cdot Y_{2 j}+\cdots+V_{p} \cdot Y_{p j}, \text { for } 1 \leq j \leq p .
$$

A multi-port structure is generally represented as in figure $1 \mathrm{a}$, where the sink ports are "linearized" by capacitive loads. The voltage across sink ports is in reality determined only by the active port voltages. In figure $1 \mathrm{~b}$, the electrical structure of the netlist is modified such that when a port is passive, the voltage across that port is zero. This is effectively done by including the port load into the interconnect and by defining the sink port as a short circuit. In this case, the port equations will be different at the sink ports from the driver ports. For a driving port $a$ and a sink port $m$, the port equations are:

$$
\begin{gathered}
I_{a}(s)=V_{1} \cdot Y_{1 a}+V_{2} \cdot Y_{2 a}+\ldots+V_{d} \cdot Y_{d a}, \\
\text { for } 1 \leq a \leq d \text { drivers only, } \\
V_{m}(s)=V_{1} \cdot H_{1 m}+V_{2} \cdot H_{2 m}+\ldots+V_{d} \cdot H_{d m}, \\
\text { for } d \leq m \leq p \text { sinks only. }
\end{gathered}
$$

\section{BUILDING THE MULTIPORT MACROMODEL}

Each transfer admittance (or voltage transfer) term is described in terms of circuit poles and residues:

$$
Y_{j k}=\sum_{i=1}^{N} \frac{f_{j k}^{i} s+g_{j k}^{i}}{h^{i} s^{2}+k^{j} s+1} .
$$

A very important observation is that the poles apply to the linear network as a whole and only the residues are specific to each transfer admittance (voltage transfer) term. Using the expression of the admittance from (1.4), and knowing 


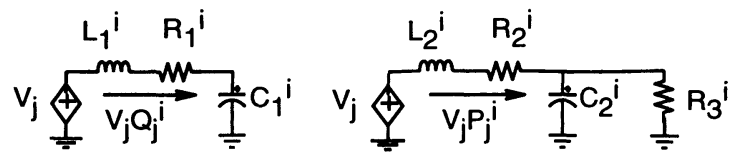

a)

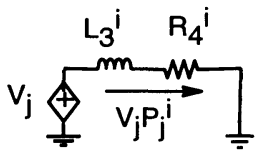

b)

Figure 2 The synthesis of the pole component: a) the two admittances for a pair of complex conjugate poles $b$ ) the admittance for a real pole.

that only $d$ ports are driving ports and that all the sink port loads are known and present in the circuit, we can rewrite the $a$-th port equation as:

$$
I_{a}(s)=\sum_{j=1}^{d} V_{j} \cdot \sum_{i=1}^{N} \frac{f_{j a}^{i} s+g_{j a}^{i}}{h^{i} s^{2}+k^{i} s+1} .
$$

Equation ( 1.5) can be rewritten as:

$$
\begin{aligned}
I_{a}(s)= & \sum_{j=1}^{d}\left(V_{j} \cdot \sum_{i=1}^{N} q_{j a}^{i} \cdot \frac{m^{i} s+n^{i}}{h^{i} s^{2}+k^{i} s+1}+r_{j a}^{i} \cdot \frac{p^{i} s}{h^{i} s^{2}+k^{i} s+1}\right) \\
= & \sum_{j=1}^{d}\left(\sum_{i=1}^{N} q_{j a}^{i} \cdot \frac{\left(m^{i} s+n^{i}\right) \cdot V_{j}}{h^{i} s^{2}+k^{i} s+1}+r_{j a}^{i} \cdot \frac{p^{i} s \cdot V_{j}}{h^{i} s^{2}+k^{i} s+1}\right) \\
= & \sum_{j=1}^{d}\left(\sum_{i=1}^{N} V_{j} \cdot P_{j}^{i} \cdot q_{j a}^{i}+V_{j} \cdot Q_{j}^{i} \cdot r_{j a}^{i}\right) \\
& P_{j}^{i}=\frac{m^{i} s+n^{i}}{h^{i} s^{2}+k^{i} s+1} \text { and } Q_{j}^{i}=\frac{p^{i} s}{h^{i} s^{2}+k^{i} s+1}
\end{aligned}
$$

In the case of a driving port, we need to determine the effect of other drivers on our port. From ( 1.5 ), the contribution of another driving port $j$ to the total current of port $a$ is a current $I_{j a}=V_{j} \cdot Y_{j a}$. Inspecting (1.6) we can see that each current contribution can be split by poles and each pole current contribution can be described using a current controlled current source. The control current is $V_{j} \cdot P_{j}^{i}$ or $V_{j} \cdot Q_{j}^{i}$ and the scalar multiplier is $q_{j a}^{i}$ or $r_{j a}^{i}$, respectively. The reason why the poles are split into two components, see (1.7), is that each sub-component can be synthesized in the form of an input admittance. The synthesized RLC structures used for the two sub-components of a complex pole pair and the component of a real pole are shown in figure 2. The control currents $V_{j} \cdot P_{j}^{i}$ and $V_{j} \cdot Q_{j}^{i}$ are the currents flowing through these simple RLC synthesized structures driven by a voltage source $V_{j}$. The values for the synthesized elements are determined using only the pole values (1.8). The $\alpha$ coefficient in (1.8) is any number greater than 1 such that $C_{2}^{i}$ has 
a real positive value.

$$
\begin{aligned}
& \text { choose } \quad \alpha>1.0 \\
& R_{1}^{i}=1 \quad R_{2}^{i}=1 \quad R_{3}^{i}=\max \left\{1, \alpha \cdot\left(\frac{4 h^{i}}{\left(k^{i}\right)^{2}}-1\right)\right\} \quad R_{4}^{i}=1, \\
& C_{1}^{i}=k^{i} \quad C_{2}^{i}=\frac{k^{i} \cdot\left(R_{2}^{i}+R_{3}^{i}\right)+\sqrt{\left(k^{i}\right)^{2} \cdot\left(R_{2}^{i}+R_{3}^{i}\right)^{2}-4 h^{i} \cdot\left(R_{2}^{i}+R_{3}^{i}\right)}}{2 R_{3}^{i}} \\
& L_{1}^{i}=\frac{h^{i}}{k^{i}} \quad L_{2}^{i}=\frac{h^{i} \cdot\left(R_{2}^{i}+R_{3}^{i}\right)}{C_{2}^{i} \cdot R_{3}^{i}} \quad L_{3}^{i}=k^{i} .
\end{aligned}
$$

The $m, n$ and $p$ coefficients are determined using the $\mathrm{R}, \mathrm{L}$, and $\mathrm{C}$ elements computed in (1.8):

$$
\begin{aligned}
\frac{m^{i} s+n^{i}}{h^{i} s^{2}+k^{i} s+1} & =\frac{\left(\frac{C_{2}^{i} R_{3}^{i}}{R_{2}^{i}+R_{3}^{i}}\right) s+\left(\frac{1}{R_{2}^{i}+R_{3}^{i}}\right)}{\left(\frac{L_{2}^{i} C_{2}^{i} R_{3}^{i}}{R_{2}^{i}+R_{3}^{i}}\right) s^{2}+\left(\frac{L_{2}^{i}+C_{2}^{i} R_{3}^{i} R_{2}^{i}}{R_{2}^{i}+R_{3}^{i}}\right) s+1} \\
\frac{p^{i} s}{h^{i} s^{2}+k^{i} s+1} & =\frac{C_{1}^{i} s}{L_{1}^{i} C_{1}^{i} s^{2}+R_{1}^{i} C_{1}^{i} s+1}, \\
\frac{n^{i}}{k^{i} s+1} & =\frac{\frac{1}{R_{4}^{i}}}{\frac{L_{3}^{i}}{R_{4}^{2}} s+1} .
\end{aligned}
$$

The $q$ and $r$ coefficients are determined such that the original residues are matched:

$$
q_{j a}^{i}=\frac{f_{j a}^{i}}{m^{i}} \quad \text { and } \quad r_{j a}^{i}=\frac{g_{j a}^{i}-f_{j a}^{i} \cdot \frac{n^{i}}{m^{i}}}{p^{i}}
$$

The block level circuitry for a two-driver circuit is shown in figure 3. The controlling currents (primary signals) are determined only by the drivers. This means that it will be more efficient to synthesize and derive these primary signals $V_{j} \cdot P_{j}^{i}$ and $V_{j} \cdot Q_{j}^{i}$ in a driver auxiliary circuitry rather than into the sink circuitry. These signals are used to model the transfer admittances at the driving ports in figure 3 as well as the transfer functions at the sink ports. The reduction in circuit size is achieved because the number of drivers is usually much smaller than the number of sinks, especially in large nets like clock distribution networks.

For each sink voltage given by (1.3) we have a set of voltage transfer functions, each corresponding to a driver. As opposed to the case of a driver port, the primary signals $\left(V_{j} \cdot P_{j}^{i} \cdot q_{j}^{i}\right.$ and $\left.V_{j} \cdot Q_{j}^{i} \cdot r_{j}^{i}\right)$ are now voltages and the sink voltage is obtained using a chain of current controlled voltage sources. Note that the components $V_{j} \cdot P_{j}^{i}$ and $V_{j} \cdot Q_{j}^{i}$ are the same as the components of 

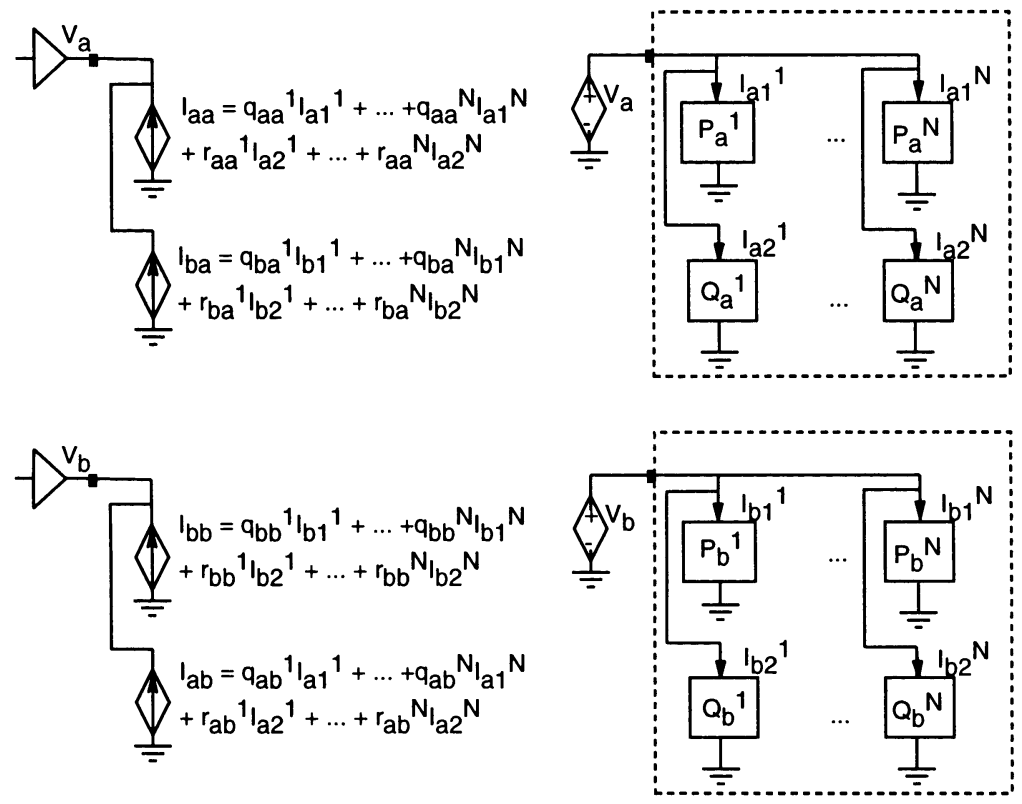

Figure 3 The block level circuitry for a circuit with two-drivers ( $a$ and $b$ ). Each driver has a driver auxiliary circuit where the primary signals are generated.
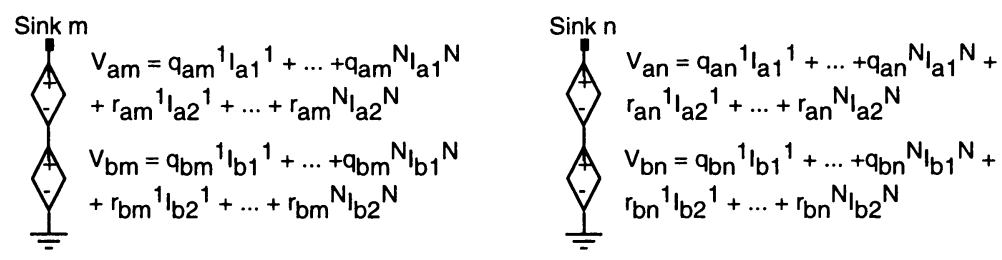

Figure 4 The structure of the sink circuitry in the case of a two-driver ( $a$ and $b$ ) two-sink ( $m$ and $n$ ) circuit.

(1.6) so the sink ports use the same partial responses from the driver auxiliary circuits. The difference is that the scalar coefficients $q$ and $r$ are, in the sink case, acting as resistances. As outlined in the previous paragraph, the sink response becomes just a linear composition of driver dependent partial responses and this eliminates any simulation that has to be done in the sink circuitry. For a circuit with two drivers ( $a$ and $b)$ and two sinks ( $m$ and $n)$, the circuitry of the sink ports is shown in figure 4.

The size of the macromodel is a function of the number of drivers $(d)$, the accuracy (number of poles required $(2 N))$ and the number of sinks $(p-d)$ :

1. resistors: $\leq 3 d N$ (equality when all poles are complex),

2. capacitors: $2 d N+p-d$, 
3. inductors: $\leq 2 d N$,

4. voltage controlled voltage sources: $d$,

5. voltage controlled current sources: $d$,

6. general format current sources: $d^{2}$ (each source is a linear combination of voltages - at drivers) [MCSpice 95],

7. general format voltage sources: $d p-d^{2}$ each source is a linear combination of voltages - at sinks) [MCSpice 95].

Maximum total number of elements: $d \cdot(7 N+p+1)$.

PRIMA also provides general macromodels for linear circuits and these are the most compact synthesized circuits with a size of $\mathrm{O}(N P)$. But the disadvantage of PRIMA's approach is that all the nodes of interest must be considered as ports in the multiport structure. In our case, the number of elements is $\mathrm{O}(d P)$ when we are building a full macromodel with all the sinks present in the circuit. The most practical approach is to build a macromodel only for the active drivers (in which case the size of our macromodel is $\mathrm{O}(d N)$ ), simulate this circuit and save the partial responses $\left(V_{j} \cdot P_{j}^{i}\right.$ and $\left.V_{j} \cdot Q_{j}^{i}\right)$ on disk and post-process them later in order to obtain the sink responses. Note that, in this approach, the macromodels that we are building become more advantageous than PRIMA's macromodels because, normally, $d \ll P$. Our macromodels are currently synthesizing the same set of $N$ pairs of poles for each driving port. In reality the synthesized circuit can be much more compact, with $N$ pairs of synthesized poles for all the driving ports. But, from an implementation point of view, this requires much more pre-processing work and makes the macromodel structure much more complicated and less intuitive.

\section{THE SIMULATION OF A MACROMODEL STRUCTURE}

In this section we present some considerations on the simulation speed of the macromodels built using our approach. Because the synthesized macromodel uses decoupled pole representations, it has the great advantage that the structure of the circuit admittance matrix is very sparse. All the matrix entries that correspond to the synthesized auxiliary circuits will be ordered in a block diagonal sub-matrix as in figure 5. All the constant coefficients used for controlled sources will be present in two off-diagonal blocks. All the driver po:ts will generate a sub-matrix that is an identity matrix because there are no direct connections between the active ports. From the general structure of the matrix, it is apparent that such a structure is very convenient for matrix LU factorization which is the most time-consuming part of a simulation. This is the reason why, sometimes, macromodels may be easier to analyze than the initial 


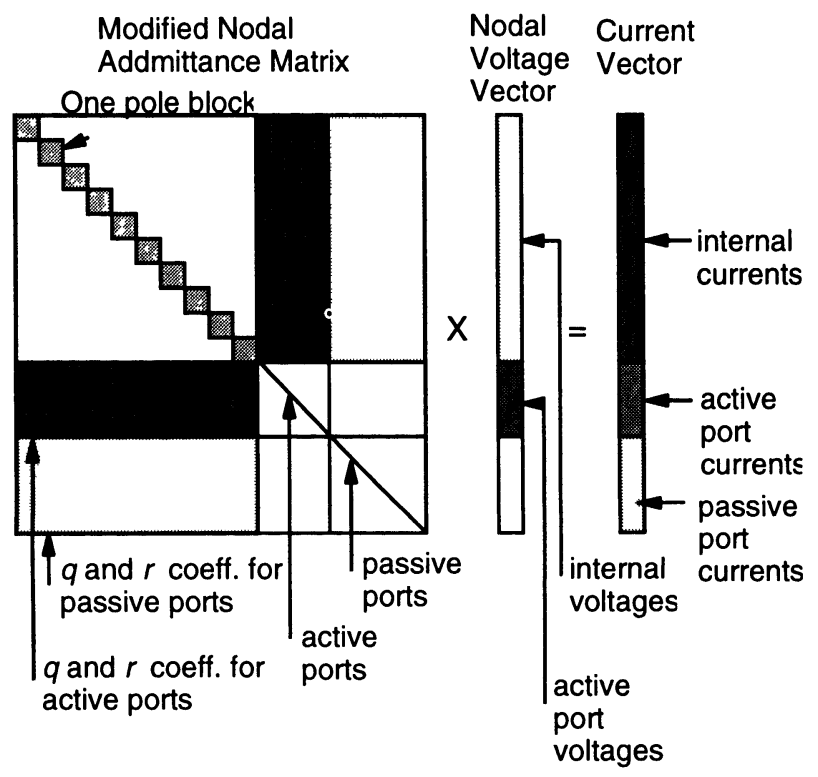

Figure 5 The macromodel admittance matrix has a very well organized structure which is convenient for matrix factorization.

circuit even if they have more components. In normal interconnect circuitry, because of the tight spacing between on-chip wires, there is a lot of capacitive and mutual inductive coupling and that results in less sparse admittance matrices. As a consequence, the MNA matrices corresponding to these nets are more dense and the use of macromodels becomes very advantageous from the simulation time point of view.

As described in the previous section, in a practical implementation, the coefficients corresponding to the passive ports are not present in the MNA matrix. These coefficients are used later in the post-processing stage in order to determine the sink responses.

\section{RESULTS}

In this section we present some results which demonstrate the accuracy and the efficiency of our approach. This macromodeling technique has been implemented in a program called Macrosim, which is primarily used for the design of very large clock networks at the Somerset Design Center. The tests performed on the tool have shown excellent accuracy (50\% delay comparison and $10 \%$ to $90 \%$ rise time comparison) with an average of less than $1 \%$ error ( $2 \%$ maximum). The simulation time speed-up achieved varies from $3 \times$ to $30 \times$ over the full netlist. 
In order to illustrate the accuracy and the speed-up of our method, we have chosen a section of the clock distribution network of one of our microprocessors. The clock section has one driver port and 370 sink ports and has an electrical model of 27484 elements ( 15148 capacitors, 7413 resistors and 4923 inductors). In table 1 we present the run times for the full netlist and for the macromodels. The macromodels are run in two different cases: in the first case, all the sinks are present in the macromodel and no post-processing is needed, and the second case, when no sinks are present in the macromodel and post-processing is needed. For the second case, the two numbers indicate the run time of the SPICE simulation and the run time of the post-processor stage. The driver of the clock section has 40 NMOS and 70 PMOS transistors, and 112 capacitors.

Table 1 Run-times for the full clock section netlist and its corresponding macromodels

\begin{tabular}{ccc}
\hline full netlist & macromodel with all sinks & macromodel with no sinks \\
\hline $434.88 \mathrm{~s}$ & $66.11 \mathrm{~s}$ & $8.94 \mathrm{~s} / 7.32 \mathrm{~s}$ \\
\hline
\end{tabular}

In figure 6 the signals at the output of the clock section driver and at one of the sink ports is shown. The macromodels used in this example (and in the normal tool flow) have 10 poles which are enough for our accuracy requirements. Note that the signal at the output node of the driver ( $a d r v$ on the plot) is very accurately captured by the macromodel $(b d r v)$ despite the fact that, due to the inductance of the lines, there is a reflected wave that produces a voltage spike.

The use of Macrosim proved to be very appealing to the clock network designers because, although it is not as fast as the static timing analysis, it is much faster than the full SPICE simulation and gives the user the flexibility to analyze the drivers separately or to monitor only some sink nodes of interest. In table 2 we show the accuracy of our macromodeling technique in comparison to the typical static analysis run that is performed on clock nets. All the errors are with respect to the full netlist SPICE simulation.

Table 2 Accuracy comparison between the static timing analysis and the macromodels

\begin{tabular}{ccc} 
Error type & Static timing & Macromodels \\
\hline Avg. delay error & $1.6 \%$ & $0.6 \%$ \\
Avg. rise time error & $11.5 \%$ & $0.8 \%$ \\
Max. delay error & $3.7 \%$ & $0.7 \%$ \\
Max. rise time error & $13.5 \%$ & $2.0 \%$ \\
\hline
\end{tabular}




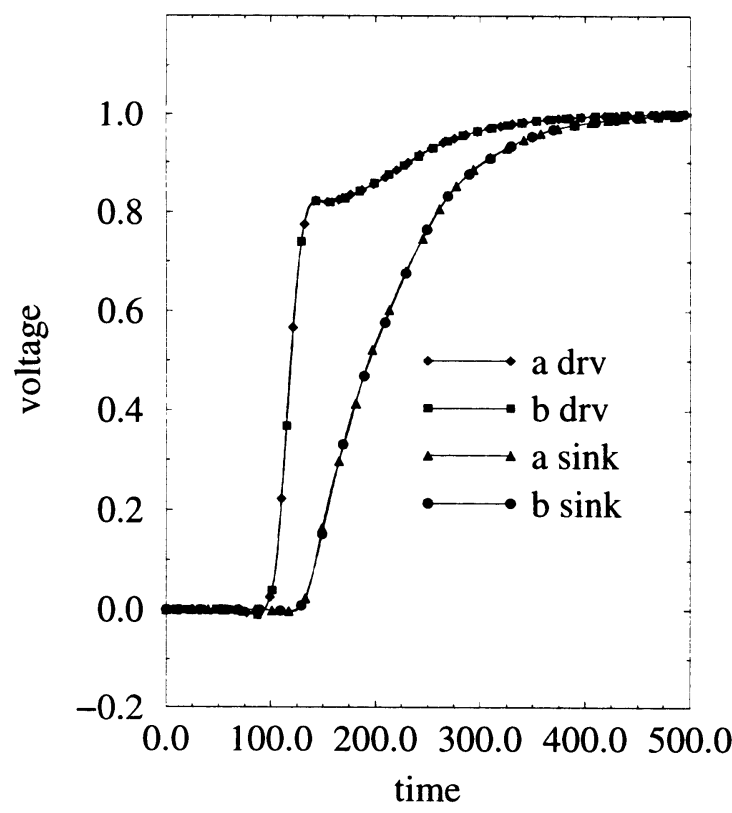

Figure 6 Simulation results for the output node of the driver $(d r v)$ and one sink node (sink). The $a$ signals are from the full network, while the $b$ signals are from the macromodel. The time and voltage units on the $\mathrm{x}$ and $\mathrm{y}$ axis have been scaled.

\section{REFERENCES}

[Kim 94] S.Y. Kim, N. Gopal and L.T. Pillage. (1994). Time Domain Macromodels for VLSI Interconnect Analysis, IEEE Transactions on CAD, vol.10, pp. 1257-1270.

[Pillage 90] L.T. Pillage, R.A. Rohrer. (1990). Asymptotic Waveform Evaluation for Timing Analysis, IEEE Transactions on CAD, vol.4, pp. 352-366.

[Dartu 96] F. Dartu, B. Tutuianu and L.T. Pileggi. (1996). RC-Interconnect Macromodels for Timing Simulation, IEEE/ACM Proceedings of DAC, pp. 544-547.

[Feldman 95] P. Feldman and R.W. Freund. (1995). Efficient linear circuit analysis by Padé approximation via the Lanczos process, IEEE Transactions on CAD, vol.5, pp. 639-649. 
304 Bogdan Tutuianu, Daksh Lehther, Madhulima Pandey, Ross Baldick

[Kerns 96] K.J. Kerns and A.T. Yang. (1996). Stable and Efficient Reduction of Large Multiport RC Networks by Pole Analysis via Congruence Transformations, IEEE/ACM Proceedings of DAC, pp. 280-285.

[Mangold 98] T. Mangold and P.Russer. (1998) A systematic approach to the problem of equivalent circuit model generation, IEEE MTT-S International Microwave Symposium Digest, Vol. 3, pp. 14091412.

[Silveira 96] L.M. Silveira, M. Kamon, I. Elfadel and J. White. (1994) A coordinate-transformed Arnoldi algorithm for generating guaranteed stable reduced-order models of arbitrary RLC circuits, IEEE/ACM Proceedings of ICCAD, pp. 288-294.

[Elfadel 97] M. Elfadel and D.D. Ling. (1997). Zeros and passivity of Arnoldi reduced-order models for interconnect networks, IEEE/ACM Proceedings of DAC.

[Odabas 97] A. Odabasioglu, M. Celik and L.T. Pileggi. (1997). PRIMA: Passive Reduced-order Interconnect Macromodeling Algorithm, IEEE/ACM Proceedings of ICCAD.

[MCSpice 95] B. Mulvaney, et. all. (1995). MCSpice - User's Manual, SSDT Circuit Design Technology, Motorola Inc.

[Valken 60] M.E. Van Valkenburg. (1960). Introduction to Modern Network Synthesis, John Wiley and Sons Inc.

[Arun 97] R. Arunachalam, F. Dartu and L.T.Pileggi. (1997). CMOS Gate Delay Models for General RLC Loading, Proceedings of ICCD, pp. 224-229. 